\title{
Detection of Plant Diseases Using Convolutional Neural Network Architectures
}

\author{
Shraddha Mahale ${ }^{a}$, Kamal Shah ${ }^{b}$ \\ Thakur College of Engineering and Technology \\ Kandivali, India ${ }^{\text {a, }} \mathrm{b}$ \\ shraddhapmahale@gmail.com ${ }^{\mathrm{a}}$, kamal.shah@thakureducation.org ${ }^{\mathrm{b}}$
}

\begin{abstract}
Plant diseases will wreak havoc on agricultural products' quality and quantity. It is important to recognize plant pathogens early on for the sake of global health and well-being. Deep learning's popularity in machine vision has recently inspired many researchers to improve the performance of plant disease detection systems. Unfortunately, most of these studies relied on AlexNet, GoogleNet, and other similar structural design rather than more recent deep designs. Furthermore, the research did not employ deep learning visualization techniques, which classify deep classifiers as "black boxes" due to their opacity. We used these three learning techniques to assess various state-of-the-art Convolutional Neural Network (CNN), AlexNet, and VGG16 architectures on a public dataset for plant disease classification in this article. In comparison to other designs, the VGG16 outperforms state-of-the-art findings in plant disease classification, with an accuracy of 97 percent. In addition, we have suggested the use of saliency maps as a means of visualizing and interpreting the CNN classification mechanism. This method of visualization improves the clarity of deep learning models and provides further insight into plant disease symptoms.
\end{abstract}

Keywords: Plant disease, Classification, Deep learning, Alexnet, VGG16, Convolutional neural network

\section{Introduction}

One of the most important sectors of the Indian economy is agriculture. It is the backbone of the country's production. Finding diseases in plants is one of the main issues facing the agriculture industry. In the past, only qualified physicians were able to diagnose illnesses. It is very difficult to reach experts for framers in remote locations. Changes in the atmosphere is one of the major factors of plant disease. If the disease is not identified in a timely manner, the big farm owner may suffer a significant loss. There are many service centers also available for the help of farmers, but it is not possible to predict them on mobile at the time of huge loss of agricultural field. Thus, sometimes they give erroneous solution for the framers and farmers felt helpless and faced more loss in production [1]. The issue of successful defense of plant diseases is closely linked to the problem of sustainable agriculture. Untried use of pesticides will contribute to the creation of long-term resistance of pathogens, thus severely reducing the capacity to fight back. Initially, people will follow specialist instructions to detect diseases and avoid them, but this procedure takes a long time and is very costly to identify diseases in a wide area. Machine Learning is a branch of computer science in which a system is given pre-data and told to learn on its own and use its learned model to complete an assignment [2]. 
Several machine learning-based models for disease diagnosis have previously been introduced, which take less time to train and identify, but these methods have certain drawbacks. Limited data sets are used in machine learning methods, which leads to overfitting; additionally, these programs are not completely automated because feature extraction, often known as handcrafted characteristics, requires the assistance of an expert [3]. Deep Learning (DL) techniques, which rely on a broad dataset to solve the problem of overfitting, and Deep Learning-based models can retrieve similar features on their own, have overcome these disadvantages. Machine learning includes DL as a subfield, that consists of several interconnected computational elements (neuron) that work together to solve problems. Deep learning has been successfully applied to bioinformatics, agriculture, and drug design, among other areas [2,3] DL reduces conventional machine learning algorithms by reducing the computational time, error ratio and it achieves a high rate of precision in the task of classification. Primary the Main aim behind our work is to identify plant diseases, and with the aid of offering solutions to recover from diseases, about the CNN model [4].

\subsection{Literature Review}

D. Das, M. Singh, S. Swaroop and Mohanty and S. Chakravarty et al. (2020) conducted through investigation in Agriculture. Discuss how to classify plants infections using Machine learning approaches like SVM, Logistic regression and Random forest. The most optimal texture properties are extracted using the Haralick algorithm. They achieve $87.6 \%$ accuracy. Here the data used is in the CSV format for the classification of Tomato disease [1].

Sachin D. Khirade et al, in their paper [2] they captured plant leaf image and processed to determine the health status of the plant. They used the SVM, ANN techniques to determine the diseases in plants. The algorithm used in these papers are SVM, back propagation and feature map. Out of them SVM worked efficiently. Image processing steps are included in their experiment.

G. Madhulatha; O. Ramadevi et al. (2020) in their paper they detect manifestation of plant diseases and classifies them into different diseases. The use of DL techniques classifies diseases. They achieved accuracy of $96.50 \%$. The model's success as an early warning or warming tool is validated by this accuracy figure. To identify the various plant diseases collected from the Plant Village dataset, the proposed approach employs a CNN model. The AlexNet design would classify the different types of plant diseases into 38 different categories [3].

S.Santhana Hari, Mr. M.Sivakumar et al. (2019) they made a model for plant disease detection for varieties of plants. The technology used here is CNN for the classification purpose. There are two conditions for datasets testing field, and under lab condition, it means the data which tested under lab condtion gives better accuracy then the data tested in the field condition. Their model gives $86 \%$ accuracy [4].

G. Shreshtha, M. Das et al. (2019) CNN is successfully applied to train the machine. With no overfitting, the accuracy percentage the test sample is $88.80 \%$. Even if the remaining 12.20 percent is filled, there is also space for improvement. This work can be applied to the agricultural domain and used to assist 
persons in monitoring their residence plants as well as farmers in keeping track of their crop. This thesis could be expanded upon to create an interface that would also include information about how to treat plant disease [5].

S.Yegneshwar Yadhav et al. (2020) their model was implemented in tensorflow back-end system to identify diseases in plants. They also implemented using Raspberry pi in open cv. Their proposed model gives $95 \%$ accuracy. The affected area percentage was calculated using the K-means clustering algorithm in MATLAB, and the optimal number of fertilizers to increase crop yield was suggested [6]. S. Arya, Rajeev Singh et al. (2019) discussed the use of AlexNet architecture and Basic CNN architecture to detect diseases in Mango and Potato leafs. It achieves accuracy $98.33 \%$ and it says that AlexNet architecture is better than Basic CNN architecture. This model is limited for the Mango and Potato disease leaf identification [7]

M. Kawatra et al. (2020) Three separate models were developed using as a starting point, AlexNet The Convolutional Neural Network (CNN) is a form of neural network that, in the technique suggested in this article. Simple AlexNet architecture is used in the first model. The optimizer AdaMax produces the best results on this model, with a validation accuracy of 96.34 percent. As the optimizer AdaMax is used, the second model presented in this paper is a hybrid of AlexNet and the Global Average Pooling Layer, which replaces the base model's last three penultimate Fully Connected (FC) Layers. In lieu of the Dense/GAP Network, the third paradigm proposed in this paper is a hybrid of AlexNet and SVM layer. This model had a validity accuracy of 99.98 percent, which is like ideal [8].

\subsection{Problem Statement}

Farm landowners and plant caretakers (in a nursery) would profit greatly from early disease detection to deter the worst from happening to their plants and to let the person know what needs to be done ahead of time for the same to function well, preventing the worst from happening to him as well. Plant diseases are a major source of productivity and economic decline in the farming industry. The prevention of diseases is a difficult task. Diseases or signs, such as colored spots or lines, are normally visible on the leaves of a plant.

\subsection{Proposed System}

This segment proposes a technique for recognizing a plant's illnesses. Using a snapshot of a leaf and various image manipulation processes, photographs classify them as though the leaf/plant has some disease.

To achieve the precision of disease detection shown in Fig.1, the framework is divided into several stages. The four phases of our system are outlined below.

Step 1: For research, a dataset called 'Plant-Village' is imported into the archive.

Step 2: Later upload, archive would be pre-treated, including copy resizing, rescaling, and collection arrangement conversion. 
Step 3: The dataset is split into sets for training and research.

Step 4: It was decided to build a standard. Inputs to CNN are used as the training dataset and the weights are changed to correctly identify the disease.

\subsection{Proposed Methodology Architecture}

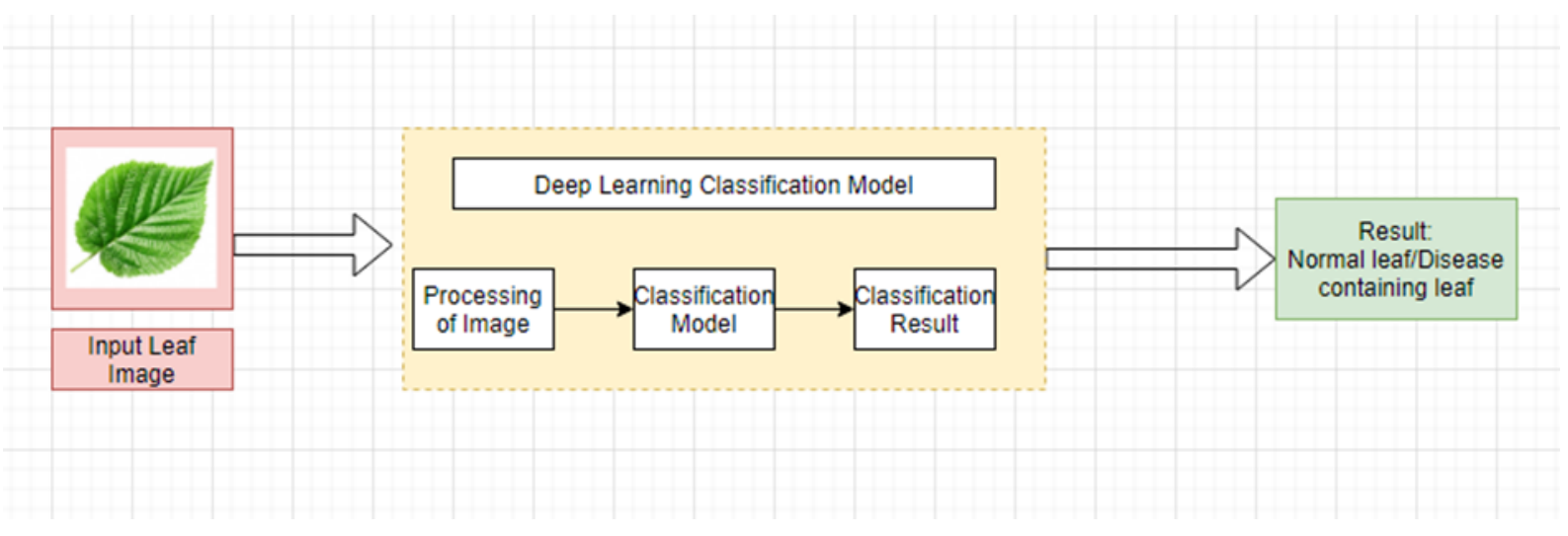

Figure 1: Block Diagram of Proposed System

In this section, the basic steps for the identification and classification of plant diseases using processing on the collected dataset are shown in the figure above.

\section{A. Acquisition}

The Images of the leaf of the plant are taken from Kaggle. This picture is in the form of RGB (Red, Green and Blue).

\section{B. Pre-processing}

Our dataset includes a variety of formats with varying resolutions because some of the files were downloaded from Plant Village. We resize the images 224X224 for CNN, AlexNet, and VGG16 architecture to improve feature extraction, reduce training time, and achieve accuracy. Figures 2 and 3 display the pre-processed photographs of mango, corn, rice, potato, and grapes.

\section{Augmentation of Data}

Augmentation of data is used to maximize the size of dataset. Some of the skills used include image rotation, affine transformation, strength transformation and perspective transformation (colour, light, and differentiate). After that, a database with 6,252, images were developed.

\section{Classification}

A validation set, a training set, and a test set were created from whole database. By dividing the 6,252, images into $70 \%$ preparation and $30 \%$ test and validation, the training range became 70 percent. The validation set is not the same as the trial set, but it is included in the testing dataset to pick parameters to avoid overfitting.

According to previous studies, segmenting the leaves from the background of the photographs and using black \& white images for classification does not boost accuracy, so this stage is skipped in the method of classification. Transfer learning, which is widely used in pre-existing neural networks, was used to detect and measure the model's accuracy 1,3,4.. The considered models have more accurate outcomes after adapting and retraining with our own training videos. Three of the experiments were performed on a computer with a 64-bit operating system, an Intel Core i3-2365M Processor, 4 GB of RAM, and a 500 GB hard disc. 


\section{Implementation}

The aim of this test is to find out whether there is a disease on the leaves in Mango, Corn, Potato, Grapes, and Rice leaves, we used three norm deep learning designs: CNN, AlexNet, and VGG16. (CNN) Convolutional Neural Network: The CNN architecture consists of a ReLU (Rectified linear unit) layer, a convolutional (conv) layer, two fully connected (FC) layers and a pooling layer, 6 ..

(Conv) Convolution Layer: Most of computation takes place in the first layer of CNN Architecture. Conv layers are feature extractors that generate a set of feature maps as they learn how to identify the features in their feedback image. Convolution and ReLU operations are carried out by this conv layer, which is accompanied by a pooling layer. The batch size influences the form of the CNN input image, channel (for RGB $=3$ and greyscale $=1$ ), width and height.

RELU: Since it conducts non-linearity in the system, the RELU layer is placed after each convolution layer. The RELU layer solves the overfitting problem and transforms all lower numbers to 0 , allowing the computer to learn faster.

Pooling layer: Because the neurons in feature maps share their weight, the pooling layer is used to reduce the spatial resolution of the feature maps. Any of the operations available in the pooling layer include normal pooling, absolute pooling, multi - resolution order less pooling, and stochastic pooling 7.

Flatten Layer: It is the layer which is used for converting 2-D data into single function vector $7 .$.

Fully Connected Layer: The neurons in this layer are fully linked to those in previous layers. The softmax algorithm is used to classify an input image using the completely connected layer, which takes a function vector as input.

The first convolution layer takes an image of size $224 \mathrm{X} 224$ as input, along with filters of size 33 and ReLU unit, and then the combining layer performs down-testing with 22 streams, resulting in a smaller function diagram. This layer's output is fed into the dual convolution layer, which convolutes the image along with a trickle size of 33 , as seen in figure 5 . The images were tweaked further, resulting in a completely connected layer that generates the output.

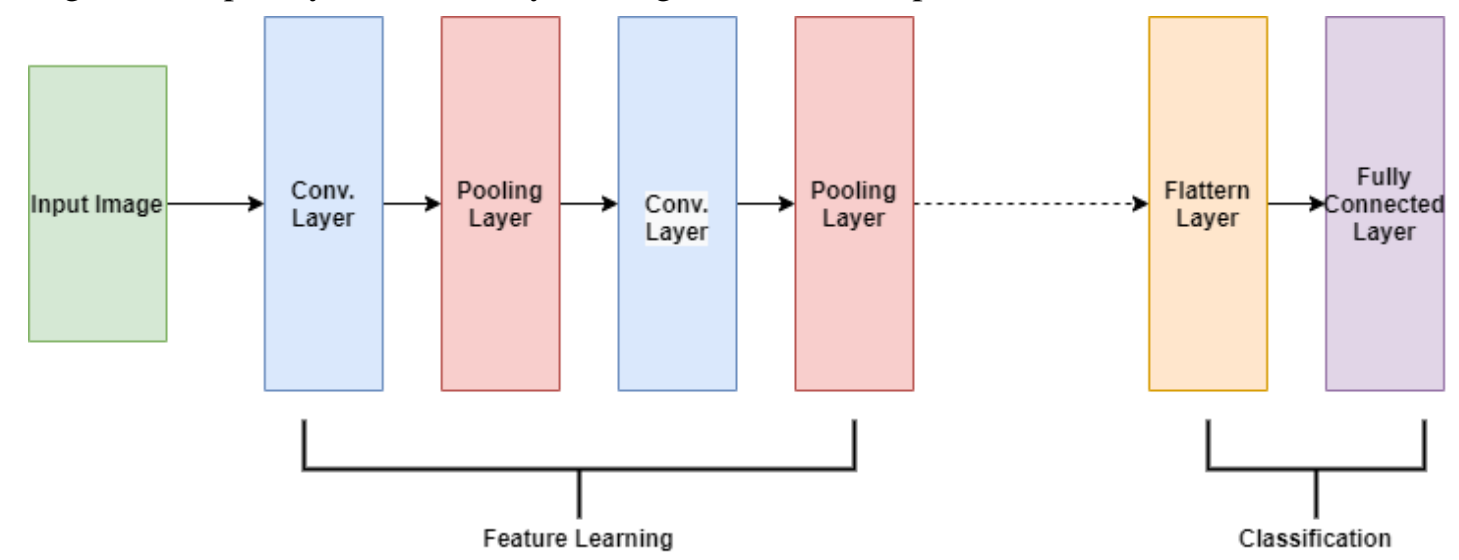

Figure 2: Architecture of Convolutional Neural Network

\section{AlexNet Architecture:}

Alex Krishevesky, et al 7., who achieved the 2012 ILVRC, designed the AlexNet architecture. There 
are eight layers in the Alexnet, each with its own set of parameters that can be learned. The model is made up of five layers, each of which uses Relu triggering, except for the output layer, which uses a combination of max pooling and 3 totally linked layers. They discovered that using the RELU as an initialization mechanism almost six-fold intensified the training process. They have used dropout layers to avoid overfitting their model. The ImageNet dataset is also used to train the algorithm. There are almost 14 million photographs in the ImageNet dataset, which are divided into a thousand categories [9].

The first conv1 layer is used in this architecture, alongside the max pooling and RELU, to filter an input image of 224 X224 pixels with a 4 pixels stride using 96 filters of 11X11 dimension. Since the convolution mechanism conducts The RELU (Rectified linear unit) or activation function is applied to all output layers of convolution, including fully connected layers, and converts all negative activation values to 0 because of non-linear operations. A conv2 layer with 256 kernels of 11X11 dimension filters the first conv1 layer's output. 384 cores of 33 range are used in the conv 3 and conv4 layers, while 256 kernels of 33 size are used in the fifth convolutional layer.

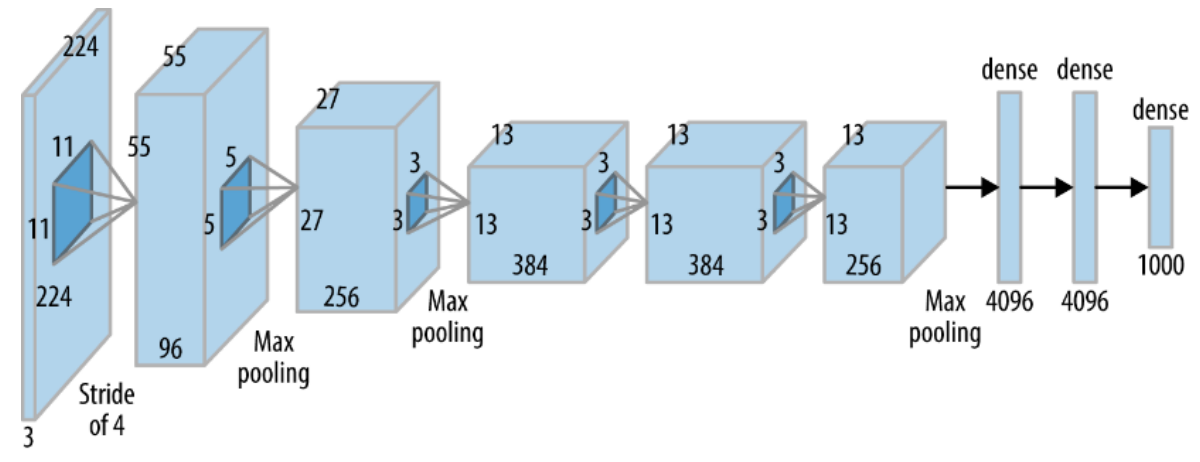

Figure 3: Architecture of AlexNet (Source: ResearchGate)

\section{VGG16:}

The network's input is a two-dimensional image $(224,224,3)$. The first two layers have the same padding and 64 channels of 3 by 3 filter size. Following a stride $(2,2)$ maxpool line, two layers of 256 stream size and filter range convolution layers are added $(3,3)$. Following that is a stride $(2,2)$ max pooling layer, which is identical to the previous layer. Following that, there are two convolution layers along with filter sizes of 3 and 3 and a 256 filter. After that, there are two adjusts of three convolution levels, as well as a max pool layer. Each has 512 filters of the same size $(3,3)$ and padding. This image is then moved to the stack of two convolution layers. In convolution and max pooling layers, filters we use is of the size $3 * 3$ instead of $11^{*} 11$ in AlexNet and $7 * 7$ in ZF-Net. It also uses $1 * 1$ pixels in some of the layers to manipulate the number of input channels. After each convolution layer, a 1-pixel padding (padding) is applied to prevent the image's spatial attribute from becoming lost. We got a $(7,7$, 512) function map after stacking the convolution and max-pooling layers. This performance is flattened to render a $(1,25088)$ function vector. Following that, there are three completely connected layers: the first takes input from the last function vector and outputs a $(1,4096)$ vector, the second layer also outputs a $(1,4096)$ vector, but the third layer outputs 1000 channels for 1000 ILSVRC challenge classes, and the output of the third fully connected layer is then transferred to the SoftMax 
layer to normalize the classification vector. The triggering mechanism for all secret layers is ReLU. ReLU is more computationally effective since it speeds up learning and reduces the chance of a vanishing gradient problem.

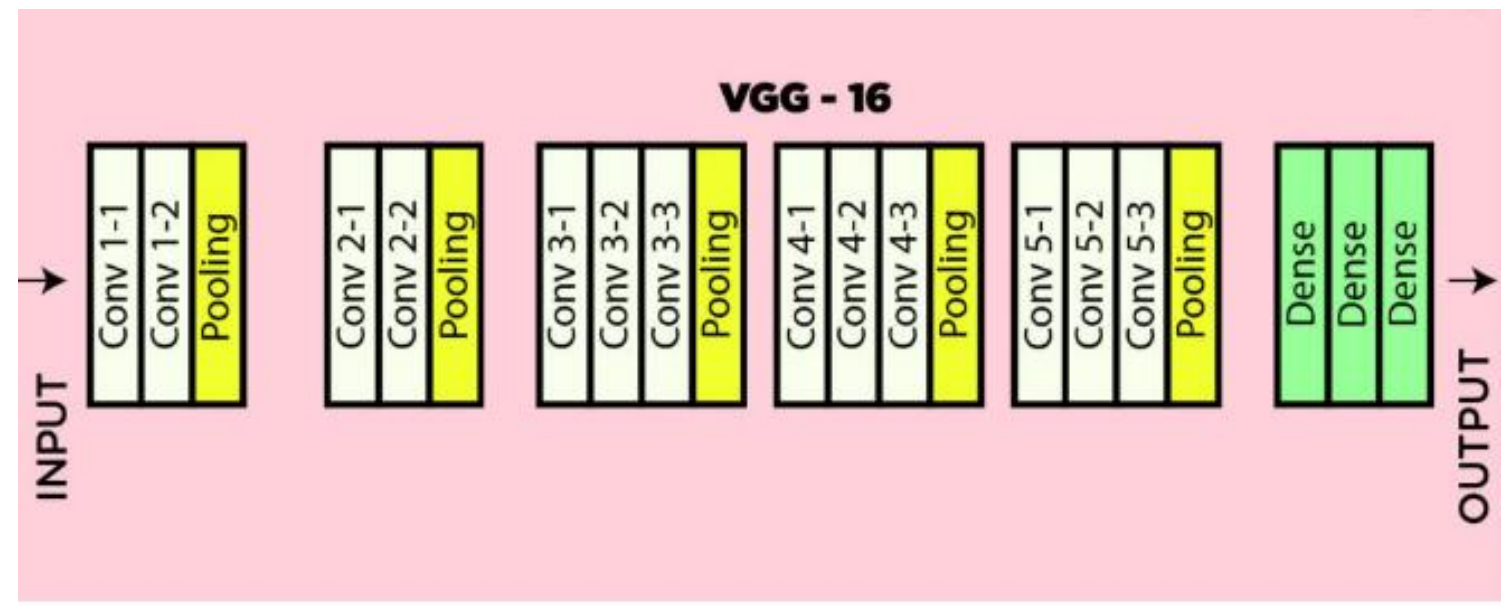

Figure 4: Architecure of VGG16(Source: geeksforgeeks.org/vgg-16-cnn-model/)

Slover Type: As a kind of solver, the SGD algorithm is used in both design and software to resolve the vanishing gradient constraint. This algorithm's main purpose is to keep the objective function from degrading (known as the sum of various functions)

Table 1 displays the Hyperparameters used in CNN, VGG16, and AlexNet designs. Hyperparameters are a kind of variable in Deep Learning is a term that refers to the hierarchical nature of a network and how it is learned.

Table 1: Hyperparameters used for training research.

\begin{tabular}{|c|l|l|l|l|}
\hline Sr.no & Hyperparameters & CNN & AexNet & VGG16 \\
\hline 1. & Slover Class & $\begin{array}{l}\text { Stochastic } \\
\text { Gradient } \\
\text { Descent } \\
(\text { SGD })\end{array}$ & $\begin{array}{l}\text { Stochastic } \\
\text { Gradient } \\
\text { Descent } \\
\text { (SGD) }\end{array}$ & $\begin{array}{l}\text { Stochastic } \\
\text { Gradient } \\
\text { Descent } \\
(\text { SGD) }\end{array}$ \\
\hline 2. & $\begin{array}{l}\text { Base Learning } \\
\text { Rate }\end{array}$ & 0.001 & 0.001 & 0.001 \\
\hline 3. & Momentum & 0.9 & 0.9 & 0.9 \\
\hline 4. & Batch Range & 128 & 128 & 128 \\
\hline
\end{tabular}

\section{Experimental Results}

We used 5032 images for preparation and research, as well as 1220 images for testing. CNN has $83.73 \%$ training accuracy, while AlexNet has an $88.56 \%$ training accuracy and VGG16 has a 97\% training accuracy. (See Figs. 5, 6, and 7). The considered model misunderstanding matrix is used to determine the test classification performance (Figure 8 and Figure 9). In the uncertainty matrix, true negative, true positive, false positive, and false negative are all represented. The higher diagonal values in the confusion matrix show the model's right estimates. 
Training and Validation Accuracy

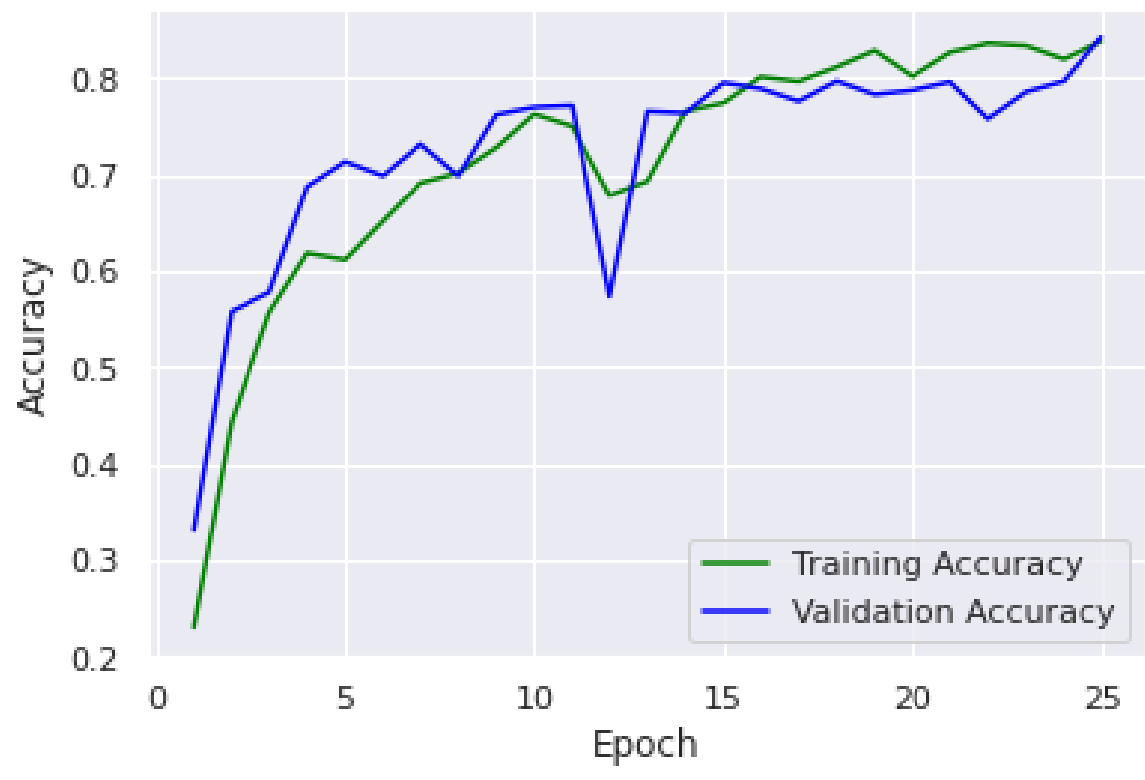

Figure 5: CNN Graph

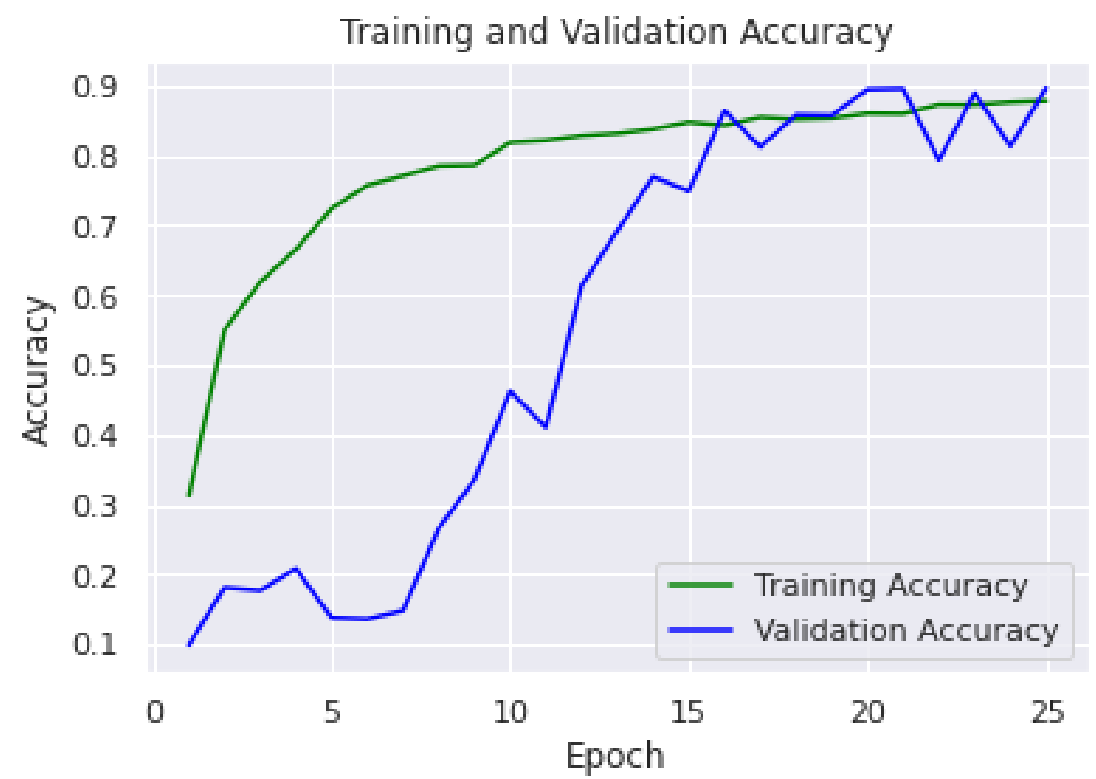

Figure 6: AlexNet Graph 


\section{Training and Validation Accuracy}

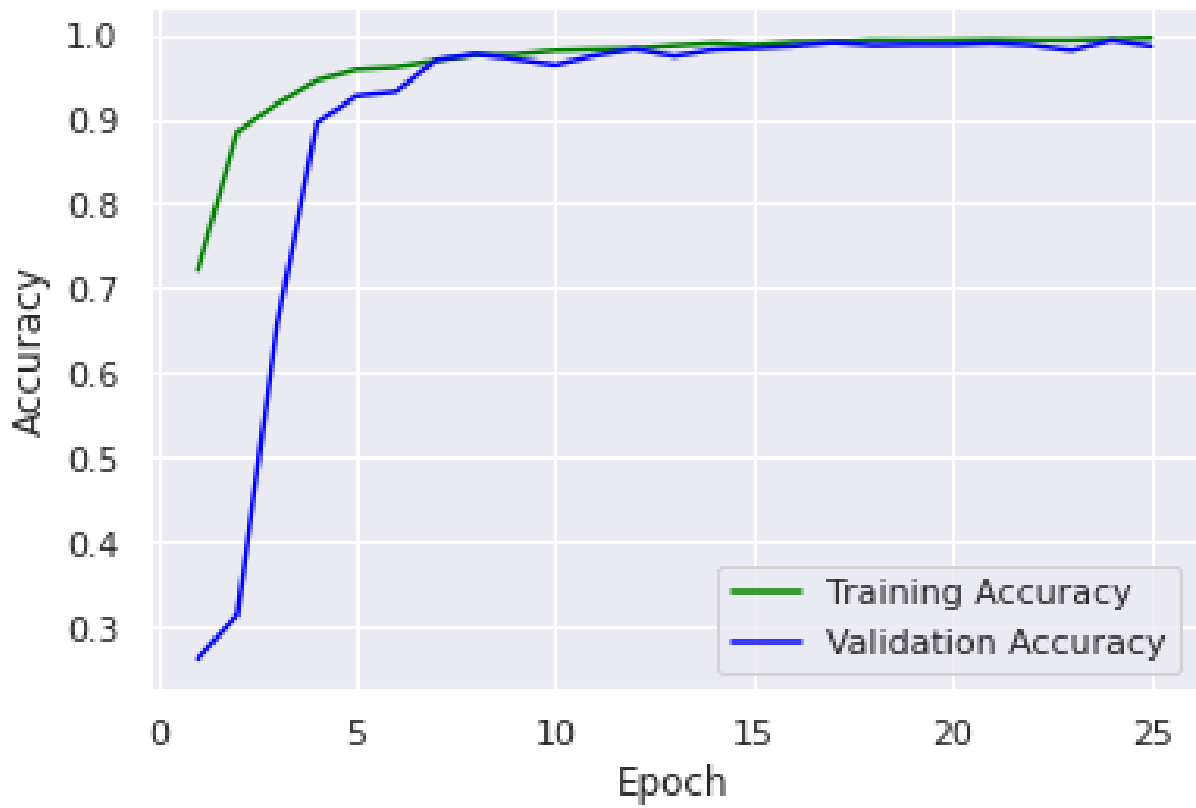

Figure 7: VGG16 Graph

Table 2: Performance Matices of CNN

\begin{tabular}{|c|c|c|c|c|}
\hline Sr. No. & Class & Precision & Recall & Accuracy \\
\hline 1. & Corn Cercospora leaf spot Gray leaf spot & 0.78 & 0.58 & \multirow{15}{*}{$83.73 \%$} \\
\hline 2. & Corn Common rust & 1.00 & 1.00 & \\
\hline 3. & Corn Healthy & 1.00 & 0.86 & \\
\hline 4. & Corn Northern Leaf Blight & 0.74 & 0.79 & \\
\hline 5. & Grape Esca Black Measles & 0.87 & 0.96 & \\
\hline 6. & Grape Leaf blight Isariopsis Leaf Spot & 0.85 & 0.92 & \\
\hline 7. & Grape healthy & 0.88 & 0.80 & \\
\hline 8. & Mango Diseased Leaf & 1.00 & 1.00 & \\
\hline 9. & Mango Healthy Leaf & 1.00 & 1.00 & \\
\hline 10. & Potato Early blight & 0.74 & 0.96 & \\
\hline 11. & Potato Late blight & 0.74 & 0.69 & \\
\hline 12. & Potato healthy & 0.86 & 0.94 & \\
\hline 13. & Rice Bacterial leaf blight & 0.60 & 0.70 & \\
\hline 14. & Rice Brown spot & 0.76 & 0.55 & \\
\hline 15. & Rice Leaf smut & 0.61 & 0.55 & \\
\hline
\end{tabular}

Table 3: Performance Matrices of AlexNet

\begin{tabular}{|r|l|c|c|c|}
\hline Sr. No. & Class & Precision & Recall & Accuracy \\
\hline 1. & Corn Cercospora leaf spot Gray leaf spot & 0.73 & 0.94 & \multirow{2}{*}{0.97} \\
\cline { 1 - 3 } 2. & Corn Common rust & 0.98 & 1.00 & \\
\cline { 1 - 3 } 3. & Corn Healthy & 0.96 & \multicolumn{2}{|c}{} \\
\hline
\end{tabular}


International Journal of Intelligent Communication, Computing and Networks

Open Access Journal (ISSN: 2582-7707)

doi.org/10.51735/ijiccn/001/19

\begin{tabular}{|c|l|c|c|}
\hline 4. & Corn Northern Leaf Blight & 0.95 & 0.76 \\
\hline 5. & Grape Esca Black Measles & 0.98 & 0.90 \\
\hline 6. & Grape Leaf blight Isariopsis Leaf Spot & 0.85 & 0.94 \\
\hline 7. & Grape healthy & 0.85 & 1.00 \\
\hline 8. & Mango Diseased Leaf & 1.00 & 0.96 \\
\hline 9. & Mango Healthy Leaf & 0.92 & 0.94 \\
\hline 10. & Potato Early blight & 0.92 & 0.74 \\
\hline 11. & Potato Late blight & 0.98 & 0.89 \\
\hline 12. & Potato healthy & 0.86 & 0.79 \\
\hline 13. & Rice Bacterial leaf blight & 0.70 & 0.78 \\
\hline 14. & Rice Brown spot & 0.69 & 0.85 \\
\hline 15. & Rice Leaf smut & 0.88 & 0.35 \\
\hline
\end{tabular}

Table 4: Performance Matrices of VGG16

\begin{tabular}{|c|l|c|c|c|}
\hline Sr.No. & Class & Precision & Recall & Accuracy \\
\hline 1. & Corn Cercospora leaf spot Gray leaf spot & 0.97 & 0.89 \\
\hline 2. & Corn Common rust & 0.98 & 0.97 \\
\hline 3. & Corn Healthy & 1.00 & 0.98 \\
\hline 4. & Corn Northern Leaf Blight & 0.99 & 0.93 \\
\hline 5. & Grape Esca Black Measles & 1.00 & 0.97 \\
\hline 6. & Grape Leaf blight Isariopsis Leaf Spot & 1.00 & 0.96 \\
\hline 7. & Grape healthy & 1.00 & 0.96 \\
\hline 8. & Mango Diseased Leaf & 1.00 & 0.98 \\
\hline 9. & Mango Healthy Leaf & 1.00 & 0.98 \\
\hline 10. & Potato Early blight & 0.99 & 0.97 \\
\hline 11. & Potato Late blight & 0.98 & 0.91 \\
\hline 12. & Potato healthy & 1.00 & 1.00 \\
\hline 13. & Rice Bacterial leaf blight & 1.00 & 1.00 & 0.91 \\
\hline 14. & Rice Brown spot & 0.98 & 0.98 & \\
\hline 15. & Rice Leaf smut & 0.92 & \\
\hline
\end{tabular}


International Journal of Intelligent Communication, Computing and Networks

Open Access Journal (ISSN: 2582-7707)

doi.org/10.51735/ijiccn/001/19

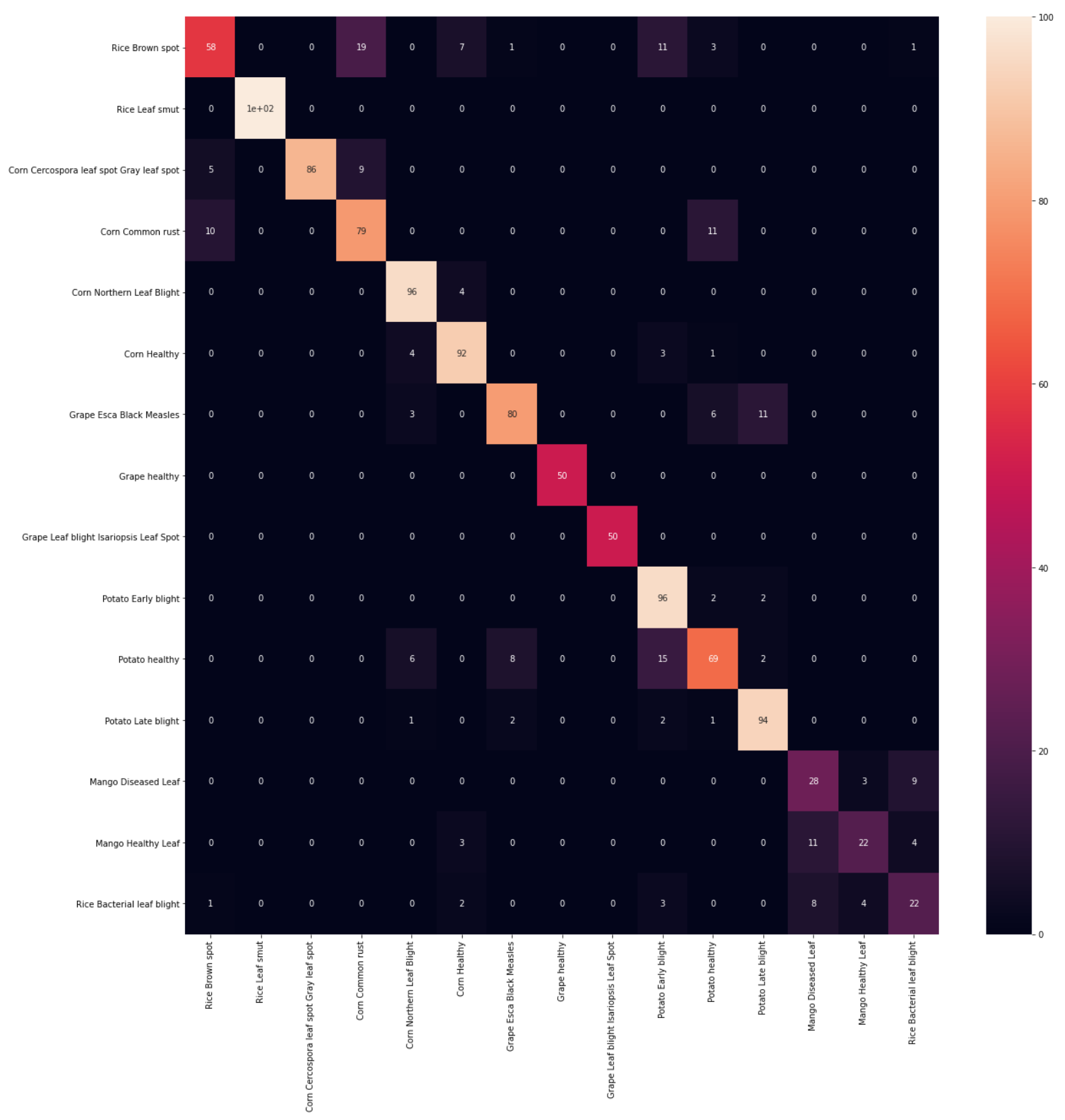

Figure 8: Confusion Matrix of $\mathrm{CNN}$ 
International Journal of Intelligent Communication, Computing and Networks

Open Access Journal (ISSN: 2582-7707)

doi.org/10.51735/ijiccn/001/19

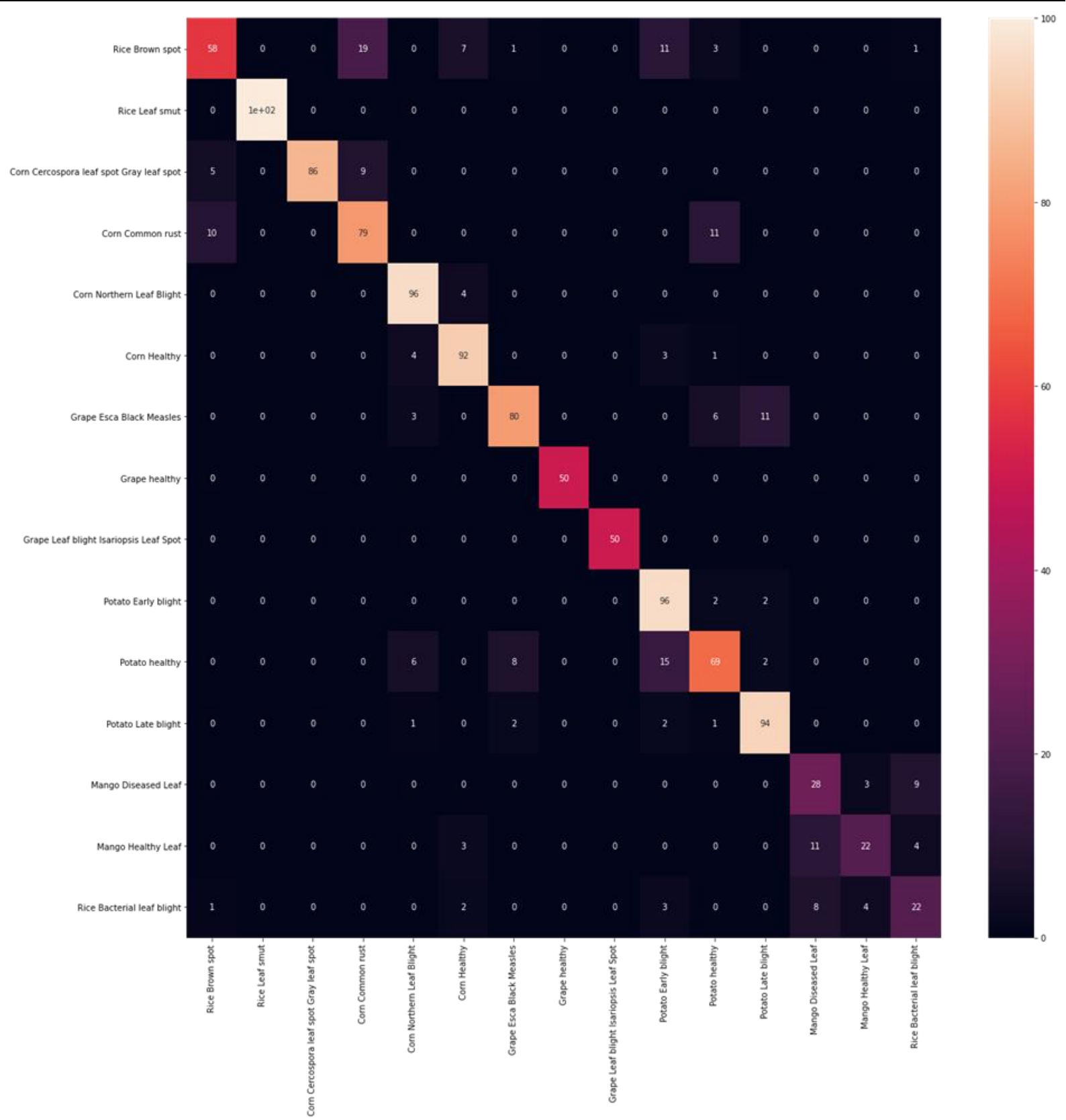

Figure 9: Confusion Matrix of AlexNet 


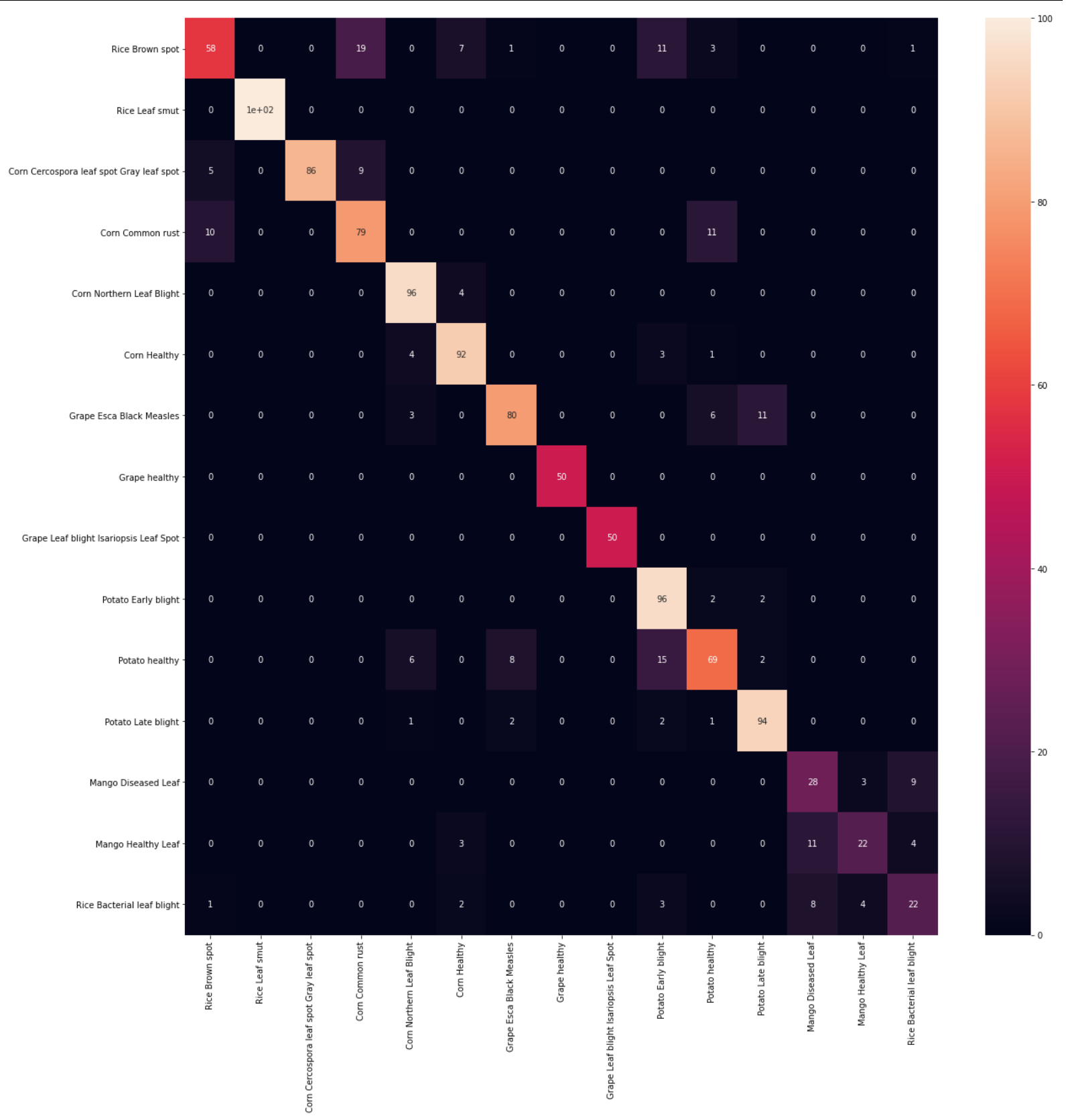

Figure 10: Confusion Matrix of VGG16

The accuracy, precision, and recall obtained from the confusion matrix obtained by CNN, AlexNet, and VGG16 Designs are described in Table-2, Table-3, and Table-4 above. As a result, VGG16 architecture achieves the best accuracy (97\%) when compared to CNN (83.73\%) and Alexnet $(88.56 \%)$. 
Accuracy \%

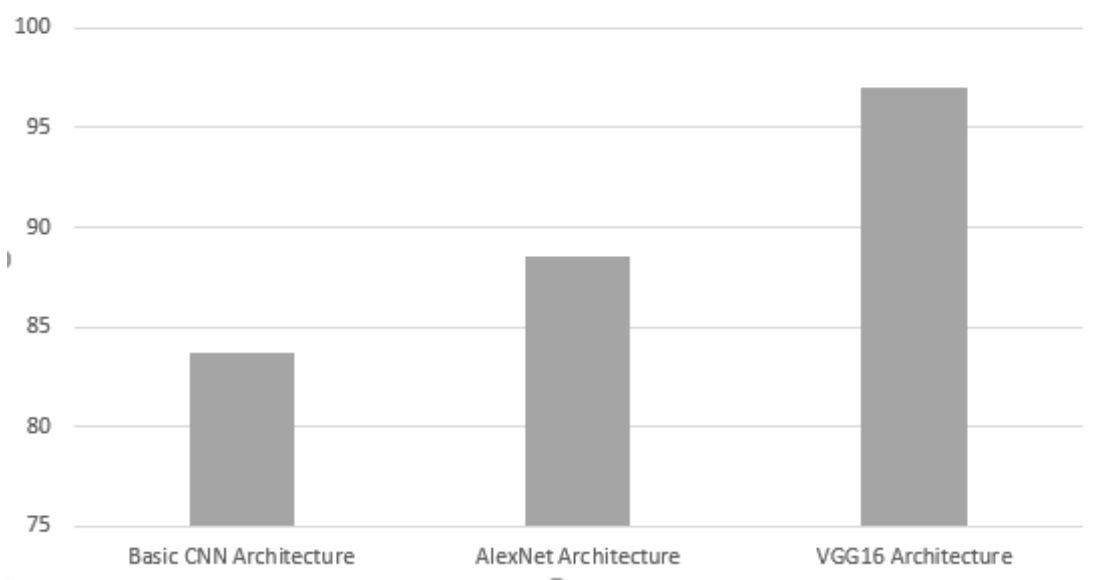

Figure 11: Comparison Between models

Fig.11 shows the comparison between models viz (Basic CNN, AlexNet, and VGG16). Hence, we have implemented our proposed system with Basic CNN, AlexNet, and VGG16 further give a prediction of Leaf passed into the system are having Disease or Not.

\section{Discussion}

The outcome of this evaluation, after classifying and comparing deep learning-based models, clearly reveals that using the VGG16 architecture will improve accuracy. VGG16 architecture hits the highest level of precision (97\%) followed by $\mathrm{CNN}$ architecture (82.73\%) and AlexNet architecture (88.86\%).

After testing, both models will forecast whether the leaf is unhealthy or steady. VGG16 design takes even longer to learn (time taken $50 \mathrm{~min}$ ) than $\mathrm{CNN}$ (time taken-20 min).

The classification accuracy of the VGG16 deep learning model is high. On the database, the model performs admirably, as shown by this result.

For the most time, numerous machine learning methods were used to find plant diseases in the early stages. The pictures are first gathered using arithmetical photographic camera. Following that, the photographs are preprocessed using various techniques. From those images, experts extract features, and give it to the classifier. The method used for image processing and feature extraction, which is a difficult and time-consuming process, determines the accuracy of classification. Deep convolutional neural networks $(\mathrm{CNN})$ have recently demonstrated a substantial increase in image segmentation and classification accuracy in many fields, including digit and natural language classification. Since it can explicitly manipulate the light, the computer vision group backed DL. CNN can identify the characteristics of images in datasets automatically. It can extract functionality and be categorized in less time using the same architecture.

Since the model can be run on a CPU without any extra hardware, unlike most neural networks, which use a lot of computing resources, such as a GPU to speed up the training process, the implementation process also requires very little hardware. 
This is since CNN, AlexNet, and VGG16 models use smaller train size images and use less training parameters. As a result, these models provide a simple and effective method for detecting plant disease with comparative results.

This paper compares the disease classification of CNN, AlexNet, and VGG16 designs on mangoes, grapes, potatoes, rice, and corn leaves. In comparison to CNN and AlexNet designs, the VGG16 architecture has high accuracy and recall. Precision separates predictive positive from actual positive, while recall separates actual positive from predictive, positive, and high precision and recall mean that the classifier is generating accurate results. The next project for the research team will be to develop a smartphone app that will diagnose the disease and be useful to farmers. Farmers will photograph diseased leaves, and the mobile device will identify the issue and include instructions about how to address it. It would be very good for farmers with vast fields because it will be more efficient and less time intensive. DL designs have also been discovered to be capable of identifying essential and irrelevant features from a series of images through this research.

\section{Acknowledgments}

I would like to take time to thank my mentor, Dr. Kamal Shah, Professor and Dean, TCET, for her keen involvement, encouraging support, and relentless help of my work during the entire process of bringing this study to fruition.

\section{References}

1. D. Das, M. Singh, S. S. Mohanty and S. Chakravarty," Leaf Disease Detection using Support Vector Machine,"2020, International Conference on Communication and Signal Processing, July 28 - 30, 2020, India, 978-1-7281-4988-2/20/\$31.00 @2020 IEEE.

2. Sachin D. Khirade, A. B. Patil," Plant Disease Detection Using Image Processing," 2015 International Conference on Computing Communication Control and Automation.

3. G. Madhulatha, O. Ramadevi," Recognition of Plant Diseases using Convolutional Neural Network," Proceedings of the Fourth International Conference on I-SMAC (IoT in Social, Mobile, Analytics and Cloud) (I-SMAC), IEEE Xplore Part Number:CFP20OSV-ART; ISBN: 978-1-7281-5464-0.

4. S.Santhana Hari, Mr. M.Sivakumar," detection of plant disease by leaf Image using convolutional neural Network," 2019 International Conference on Vision Towards Emerging Trends in Communication and Networking (ViTECoN), 978-1-5386-9353-7/19/\$31.00 @2019 IEEE.

5. G. Shreshtha, M.Das,"Plant Disease Detection Using CNN", Proceedings of 2020 IEEE Applied Signal Processing Conference (ASPCON), ISBN: 978-1-7281-6882-1

6. S.Yegneshwar Yadhav, T.Senthilkumar," Plant Disease Detection and Classification using CNN Model with Optimized Activation Function." Proceedings of the International Conference on Electronics and Sustainable Communication Systems (ICESC 2020), IEEE Xplore Part Number: CFP20V66-ART; ISBN: 978-1-7281-4108-4 
7. S. Arya, R. Singh," A Comparative Study of CNN and AlexNet for Detection of Disease in Potato and Mango leaf," 2019 2nd International Conference on Issues and Challenges in Intelligent Computing Techniques (ICICT), 978-1-7281-1772-0 @2019 IEEE

8. Mihir Kawatra,Shreyas Agrawal." Leaf Disease Detection using Neural Network Hybrid Models," 2020 IEEE 5th International Conference on Computing Communication and Automation (ICCCA) Galgotias University, Greater Noida, UP, India. Oct 30-31, 2020

9. J. Richard Landis and Gary G. Koch - The Measurement of Observer Agreement for Categorical Data, Biometrics, Vol. 33, No. 1 (Mar. 1977), pp. 159-174 